\title{
Field-free orientation of molecules
}

\section{Machholm, Mette; Henriksen, Niels Engholm}

\section{Published in:}

Physical Review Letters

Link to article, DOI:

10.1103/PhysRevLett.87.193001

Publication date:

2001

\section{Document Version}

Publisher's PDF, also known as Version of record

Link back to DTU Orbit

\section{Citation (APA):}

Machholm, M., \& Henriksen, N. E. (2001). Field-free orientation of molecules. Physical Review Letters, 87(19), 193001. https://doi.org/10.1103/PhysRevLett.87.193001

\section{General rights}

Copyright and moral rights for the publications made accessible in the public portal are retained by the authors and/or other copyright owners and it is a condition of accessing publications that users recognise and abide by the legal requirements associated with these rights.

- Users may download and print one copy of any publication from the public portal for the purpose of private study or research.

- You may not further distribute the material or use it for any profit-making activity or commercial gain

- You may freely distribute the URL identifying the publication in the public portal

If you believe that this document breaches copyright please contact us providing details, and we will remove access to the work immediately and investigate your claim. 


\title{
Field-Free Orientation of Molecules
}

\author{
Mette Machholm \\ Department of Computational Science, The National University of Singapore, Singapore, Singapore 119260
}

Niels E. Henriksen

Department of Chemistry, Technical University of Denmark, DTU 207, DK-2800 Lyngby, Denmark

(Received 11 June 2001; published 19 October 2001)

\begin{abstract}
We consider impulsive excitation of a linear polar molecule by a plane polarized electromagnetic "half-cycle" pulse in the terahertz range. A rotational wave packet is created with angular momentum states of different parity. The time evolution of the wave packet corresponds to alternating molecular orientations with respect to the polarization axis of the field. This field-free time-dependent orientation of the molecule is computationally demonstrated, also at finite temperatures, with $\mathrm{LiH}$ and $\mathrm{NaI}$ as examples.
\end{abstract}

DOI: 10.1103/PhysRevLett.87.193001

Alignment and orientation of molecules can play an important role in the outcome of molecular collisions as well as in the interaction between molecules and electromagnetic fields (Refs. [1-3], and references therein). Several schemes for the alignment or orientation of molecules have been suggested (Refs. [4-10], and references therein). These schemes are based on the interaction with static or time-dependent electromagnetic fields. Molecules in intense laser fields can align, e.g., using a laser frequency on resonance with an electronic transition [10] or an off-resonant infrared field keeping the molecule in the electronic ground state [8].

Ultrashort laser pulses can generate a superposition of molecular eigenstates, i.e., a nonstationary wave packet. We consider here the excitation of angular motion, that is, the creation of a wave packet in the angular degrees of freedom via short-pulse, off-resonant excitation with respect to rotational transitions. Recently, field-free alignment, after turn-off of a laser pulse, was proposed [11,12]. Wavepacket revivals were found corresponding to strong enhancement of the alignment subsequent to the laser pulse.

Time-dependent orientation of diatomic molecules has been considered within the rigid-rotor approximation $[13,14]$. Field-free orientation was, in particular, discussed in Ref. [13]. Impulsive ("sudden") excitation generated by an ultrashort pulse was described analytically by the first term in the Magnus expansion of the propagator. Oscillatory motion (libration) in $\theta$, the angle between the polarization axis of the field and the molecular axis, was created: The wave packet corresponds to the motion of the molecule which alternates between orientations in the forward and backward hemisphere, respectively, with respect to the polarization axis of the field. The initial state was assumed to be one of the rotational eigenstates.

The key result presented in this Letter is that field-free time-dependent orientation for a molecule like $\mathrm{LiH}$ can be generated after the turn-off of a state-of-the-art electromagnetic half-cycle pulse [15]. This proposition is based on theoretical results for diatomic molecules where rotation and vibration are included. The coupling to the field
PACS numbers: $33.15 . \mathrm{Kr}, 33.80 .-\mathrm{b}$

is described within the electric-dipole approximation, and the time-dependent Schrödinger equation is solved numerically without any additional approximations.

We consider a diatomic molecule, in the electronic ground state, described by the Hamiltonian

$$
H_{0}=-\frac{\hbar^{2}}{2 m}\left[\frac{\partial^{2}}{\partial R^{2}}+\frac{2}{R} \frac{\partial}{\partial R}\right]+\frac{L^{2}}{2 I}+V(R),
$$

where $m$ is the reduced mass of the nuclei, $I=m R^{2}$ is the moment of inertia at the internuclear distance $R, L^{2}$ is the angular momentum operator, and $V(R)$ is the internuclear potential.

The molecule which is assumed to have a permanent dipole moment is subject to a flash of coherent electromagnetic radiation. The field-molecule coupling $H_{I}(t)$ is an electric-dipole coupling with a plane polarized electromagnetic field,

$$
H_{I}(t)=-\mu(R) E_{0} \cos \theta a(t) \cos (\omega t),
$$

$\mu(R)$ is the electric-dipole moment at the internuclear distance $R$, and the envelope function $a(t)$ is centered at the time $t=t_{p}$ and chosen as a Gaussian pulse shape,

$$
a(t)=e^{-\left(t-t_{p}\right)^{2} / \sigma^{2}} .
$$

The impulsive nature of the excitation implies that the pulse duration, $\sigma$, is much shorter than a rotational period $\tau$ and the excitation is, consequently, off resonant.

The total Hamiltonian is $H_{0}+H_{I}(t)$, and, with a linearly polarized pulse, the quantum number associated with the projection of the angular momentum along the polarization axis, $M$, is conserved. The wave function can be represented by the expansion

$$
\Psi_{M}(R, \theta, \phi, t)=\sum_{J^{\prime}} \chi_{J^{\prime}}(R, t) Y_{J^{\prime}, M}(\theta, \phi),
$$

where the spherical harmonics satisfy

$$
L^{2} Y_{J^{\prime}, M}(\theta, \phi)=J^{\prime}\left(J^{\prime}+1\right) \hbar^{2} Y_{J^{\prime}, M}(\theta, \phi) .
$$

The initial state is an eigenfunction of $H_{0}, \Psi_{M}(R, \theta$, $\phi, 0)=\chi_{J}^{\nu}(R) Y_{J, M}(\theta, \phi)$, where $\chi_{J}^{\nu}(R)$ is a rovibrational eigenfunction corresponding to the quantum numbers $(\nu, J)$. When this expansion in spherical harmonics is 
substituted into the time-dependent Schrödinger equation, a set of coupled equations in the radial coordinate, $R$, is obtained. The unknown wave functions $\chi_{J^{\prime}}(R, t)$ are obtained via a grid representation [16].

The orientation can, e.g., be monitored by

$$
\begin{aligned}
O_{\mathbf{n}}(t)= & \int_{\mathbf{R} \in \mathrm{U}}\left|\Psi_{M}(R, \theta, t)\right|^{2} d \mathbf{R} \\
& -\int_{\mathbf{R} \in \mathrm{L}}\left|\Psi_{M}(R, \theta, t)\right|^{2} d \mathbf{R},
\end{aligned}
$$

where $\mathbf{n}=(\nu, J, M)$ denotes the dependence on the initial state, $d \mathbf{R}=R^{2} d R \sin \theta d \theta d \phi$, "U" is the upper hemisphere $(0<\theta<\pi / 2)$, and " $\mathrm{L}$ " is the lower hemisphere $(\pi / 2<\theta<\pi)$. Note that $\max \left[O_{\mathbf{n}}(t)\right]=1$, when the "head" of the molecule is in the upper hemisphere, and $\min \left[O_{\mathbf{n}}(t)\right]=-1$, when the head is in the lower hemisphere. Alternatively, the expectation value of $\cos \theta, \quad\langle\cos \theta\rangle_{\mathbf{n}}(t)=\left\langle\Psi_{M}(R, \theta, t)|\cos \theta| \Psi_{M}(R, \theta, t)\right\rangle$, can be used as a measure of the orientation [14]. $\langle\cos \theta\rangle_{\mathbf{n}}(t) \in[-1,1]$. Note that the limiting values of $\langle\cos \theta\rangle_{\mathbf{n}}(t)$ are attained only when the orientation as well as the alignment is perfect.

In the rigid-rotor approximation, it is possible to obtain insight concerning the time evolution. The wave packet can be expressed as the time evolution of the initial state $|J, M\rangle$ during the electromagnetic pulse followed by the time evolution associated with the field-free rigid-rotor dynamics $U_{0}\left(t, t_{0}\right)=\exp \left[-i L^{2}\left(t-t_{0}\right) /\left(2 \hbar I_{e}\right)\right]$, where $t_{0}$ is the effective interaction time:

$$
\left|\psi_{M}(\theta, t)\right|^{2}=\left|\left\langle\theta, \phi\left|U_{0}\left(t, t_{0}\right) U\left(t_{0}, 0\right)\right| J, M\right\rangle\right|^{2} .
$$

The wave packet, after the field-molecule interaction, is expressed by an expansion in spherical harmonics,

$$
\left\langle\theta, \phi\left|U\left(t_{0}, 0\right)\right| J, M\right\rangle=\sum_{J^{\prime}} c_{J^{\prime}} Y_{J^{\prime}, M}(\theta, \phi),
$$

where $c_{J^{\prime}}$ depend on the initial state $|J, M\rangle$. Thus,

$$
\begin{aligned}
\left|\psi_{M}(\theta, t)\right|^{2}= & \sum_{J^{\prime}}\left|c_{J^{\prime}}\right|^{2}\left|Y_{J^{\prime}, M}(\theta, \phi)\right|^{2} \\
& +2 \sum_{J^{\prime}} \sum_{J^{\prime \prime}<J^{\prime}}\left|c_{J^{\prime}}\right|\left|c_{J^{\prime \prime}}\right| Y_{J^{\prime}, M}(\theta, \phi) Y_{J^{\prime \prime}, M}^{*}(\theta, \phi) \\
& \times \cos \left[\Delta E_{J^{\prime} J^{\prime \prime}}\left(t-t_{0}\right) / \hbar-\varphi_{J^{\prime} J^{\prime \prime}}\right]
\end{aligned}
$$

where

$$
\begin{aligned}
\varphi_{J^{\prime} J^{\prime \prime}} & =\arg \left(c_{J^{\prime}}\right)-\arg \left(c_{J^{\prime \prime}}\right) \\
\Delta E_{J^{\prime} J^{\prime \prime}}=\left(E_{J^{\prime}}-E_{J^{\prime \prime}}\right) & =B_{e}\left[J^{\prime}\left(J^{\prime}+1\right)-J^{\prime \prime}\left(J^{\prime \prime}+1\right)\right] \\
& =n\left(2 B_{e}\right), \quad \text { where } n \in Z .
\end{aligned}
$$

All the frequencies are equal to an integer times $2 B_{e} / \hbar$, where $B_{e}=\hbar^{2} /\left(2 I_{e}\right)$, which implies that revivals in the wave packet will show up at time intervals given by the fundamental period $\tau=\pi \hbar / B_{e}$. This is also the time dependence of $O_{\mathbf{n}}(t)$ and $\langle\cos \theta\rangle_{\mathbf{n}}(t)$.

In order to avoid numerical time propagation over periods of several hundred picoseconds, we use an approach similar to that in Eq. (7). We project the wave packet onto the eigenstates of $H_{0}$, after the interaction with the electromagnetic pulse, and continue the propagation using the known time evolution of the eigenstates.

Figure 1 illustrates the principle of field-free timedependent orientation. Within the rigid-rotor approximation, a superposition of the states $J^{\prime}=0$ and $\left(J^{\prime}, M\right)=(1,0)$ (with equal weights) is assumed at $t=0$. The orientation parameters are given by $O(t)=$ $(\sqrt{3} / 2) \cos \left(\Delta E_{10} t / \hbar\right)$ and $\langle\cos \theta\rangle(t)=\sqrt{1 / 3} \cos \left(\Delta E_{10} t /\right.$ $\hbar)$. Thus, at $t=0$ and $t=\tau$ the molecule is essentially found only in the forward hemisphere $(\theta<\pi / 2)$, whereas at $t=\tau / 2$ the molecule is almost exclusively positioned in the backward hemisphere. The physical basis for this effect is the interference between the two angular momentum states [see Eq. (9)], which have different parity with respect to inversion at the origin.

A rotational wave packet with rotational states of different parity can be created by the interactions with an ultrashort pulse of electromagnetic radiation, where the pulse duration is shorter than the optical period of the radiation. Such "half-cycle" pulses (HCP) can be generated experimentally [15]. The important feature of the pulse is a large asymmetry in the magnitude of the positive and the negative peak value of the electric field, e.g., 5:1 as in Ref. [15]. We model a HCP using the envelope function $a(t)$ in Eq. (3) with $\sigma=\pi /(\omega 2 \sqrt{\ln 2})$. For a peak frequency around $1 \mathrm{THz}\left(\hbar \omega=36 \mathrm{~cm}^{-1}\right)$ the temporal width is $450 \mathrm{fs}$ ( $\sigma=279 \mathrm{fs})$. Experimentally a focused field with a peak field of $E_{0}>E_{\mathrm{HCP}}=1.5 \times 10^{7} \mathrm{~V} / \mathrm{m}$ can be generated [15]. We use peak fields of $E_{0}=1,2$, or $4 E_{\mathrm{HCP}}$ in our numerical simulations.

We consider $\mathrm{LiH}$ and $\mathrm{NaI}$ in the electronic ground state, that is, two polar molecules with very different rotational constants, $B_{e} \approx 7.51 \mathrm{~cm}^{-1}$ and $0.12 \mathrm{~cm}^{-1}$, respectively,

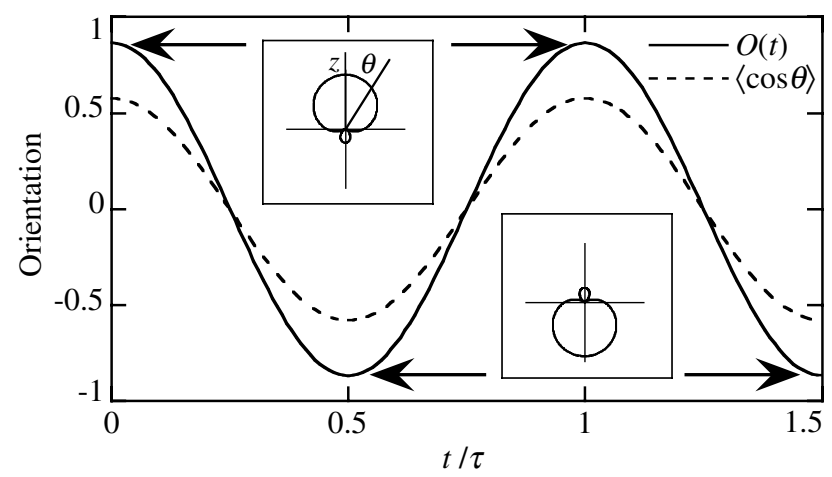

FIG. 1. The principle of field-free orientation - orientation parameters as a function of time. A superposition of the states $J^{\prime}=0$ and $\left(J^{\prime}, M\right)=(1,0)$ (with equal weights) is assumed at $t=0$, in the rigid-rotor approximation. $\tau=\pi \hbar / B_{e}$ is the fundamental rotational period. The insets show polar plots of the rotational wave function, at the times indicated by the arrows. The complete wave function is obtained by a rotation of the curves about the $z$ axis. 


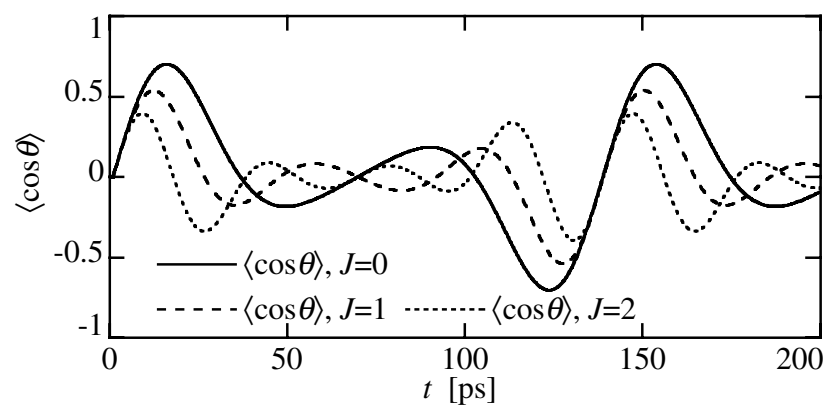

FIG. 2. The orientation parameter $\langle\cos \theta\rangle_{J}(t)$ for NaI for the initial states $J=0,1$, and 2 . For $J=1$ and 2 , the curve is the sum over $\langle\cos \theta\rangle_{(J, M)}(t)$ for all initial $M$ states. The peak field strength of the HCP is $E_{0}=3 \times 10^{7} \mathrm{~V} / \mathrm{m}$.

and the corresponding rotational periods are $\tau=2.2 \mathrm{ps}$ and $138 \mathrm{ps}$. The fundamental vibrational frequency is $1406 \mathrm{~cm}^{-1}$ and $258 \mathrm{~cm}^{-1}$, respectively. The calculations are based on the potentials $V(R)$ and dipole moments $\mu(R)$ given in Refs. $[17,18]$. The permanent dipole moments are $\mu\left(R_{e}\right)=5.88 \mathrm{D}$ and $9.2 \mathrm{D}$ for $\mathrm{LiH}$ and $\mathrm{NaI}$, respectively.

Figure 2 shows $\langle\cos \theta\rangle_{J}(t)$ for $\mathrm{NaI}$ for the initial states $J=0,1$, and 2 . A pronounced field-free timedependent orientation is observed, with full revivals spaced by $\tau=138 \mathrm{ps}$. The peak value of $\langle\cos \theta\rangle_{J}(t)$ decreases with increasing $J$. Note that the three curves are not phase shifted; therefore, revivals are also present in the sum of the curves.

The population in the angular momentum eigenstates of $\mathrm{NaI}$ and $\mathrm{LiH}$ after the impulsive excitation, for various initial states, is shown in Fig. 3. Several higher angular momentum states are populated. Selection rules, like $\Delta J= \pm 1$ and $\Delta J= \pm 2$, apply only for long pulses with

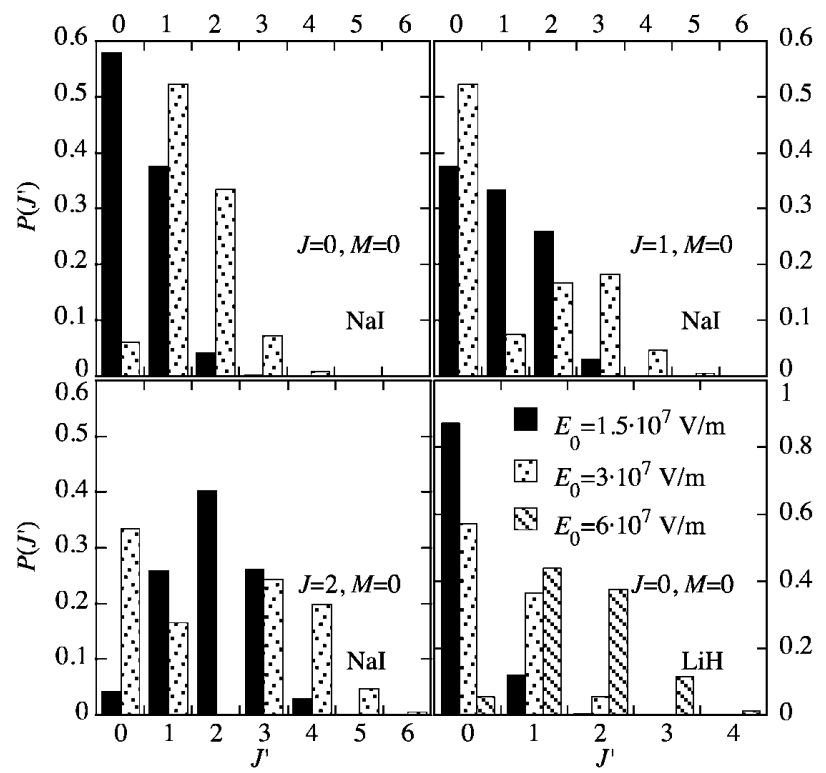

FIG. 3. The population in angular momentum eigenstates of $\mathrm{NaI}$ and $\mathrm{LiH}$ after impulsive excitation using a HCP. low or moderate intensities. When we compare these results to the results obtained within the rigid-rotor approximation, $P\left(J^{\prime}\right)$ differs by $0.01 \%-0.5 \%$ and $0.2 \%-8 \%$ for $\mathrm{NaI}$ and $\mathrm{LiH}$, respectively. The HCP used in this work induces, essentially, no vibrational excitation.

The results presented above were based on the assumption that the molecule initially was in an eigenstate of the Hamiltonian $H_{0}$. The thermally averaged orientation takes the form

$$
\langle\cos \theta\rangle_{T}(t)=\sum_{J=0}^{\infty} P(J) \sum_{M=-J}^{M=J}\langle\cos \theta\rangle_{(J, M)}(t),
$$

where a summation over vibrational quantum numbers is omitted, since we consider only low temperatures, and $P(J)$ is the Boltzmann distribution associated with the rotational states. Within the rigid-rotor approximation

$$
P(J)=\exp \left\{-J(J+1) B_{e} /\left(k_{B} T\right)\right\} / Q_{\text {rot }},
$$

where $Q_{\text {rot }}=8 \pi^{2} I_{e} k_{B} T / h^{2}$. The energy level with the highest weight in the Boltzmann average is at $J_{\max }=$ $\sqrt{I_{e} k_{B} T} / \hbar-1 / 2$ (rounded off to the closest integer). For $\mathrm{NaI}$ at $T=10 \mathrm{~K}, J_{\max }=5$ and the distribution is, in addition, fairly broad. The thermal average is a weighted sum of time-dependent terms with different oscillation frequencies and phases. Within the rigid-rotor approximation, the angular wave packet created by the pulse contains the frequencies $\nu_{n}=n\left[B_{e} /(\pi \hbar)\right]$, where $n=1,2,3, \ldots$,

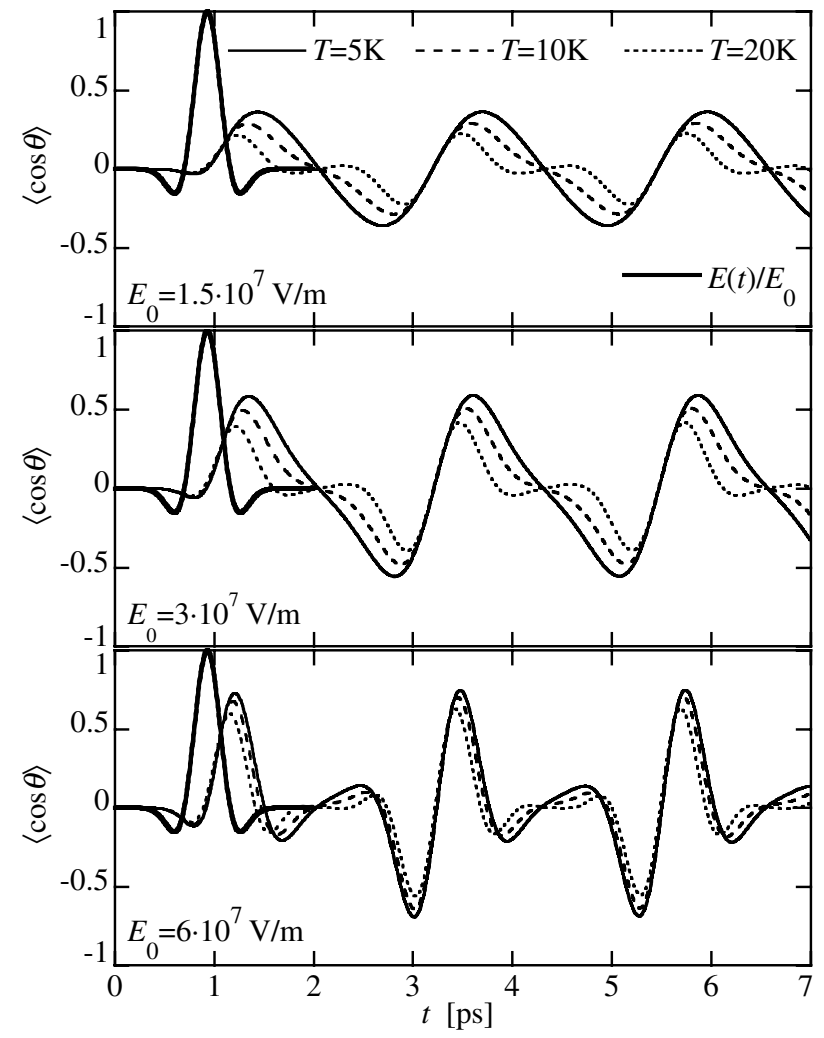

FIG. 4. The orientation parameter $\langle\cos \theta\rangle_{T}(t)$ for $\mathrm{LiH}$ at the temperatures and field strengths specified in the figure. $E(t) / E_{0}$ is the normalized electric field of the HCP. 


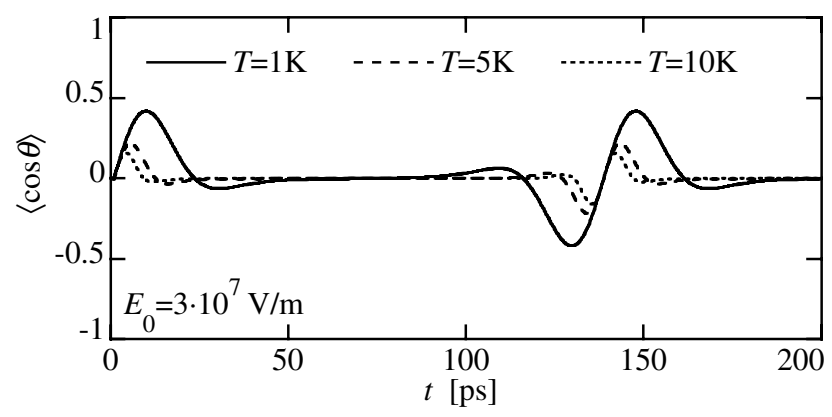

FIG. 5. The orientation parameter $\langle\cos \theta\rangle_{T}(t)$ for $\mathrm{NaI}$ at the temperatures and field strength specified in the figure.

irrespective of the initial state. Thus, we expect revivals also in the Boltzmann average, provided the phases are independent of the initial state.

Figure 4 shows the orientation parameter $\langle\cos \theta\rangle_{T}(t)$ for $\mathrm{LiH}$ at the temperatures and field strengths specified in the figure. A strong orientation effect is observed after turn-off of the pulse, with full revivals spaced by $\tau=2.2 \mathrm{ps}$. The coupling between vibration and rotation is included in the calculation, but the observed revival time is in good agreement with Eq. (9), i.e., the dynamics within the rigidrotor approximation. At the studied temperatures only initial states corresponding to $J \leq 2$ make a significant contribution.

The time-dependent orientation effect is less robust to thermal averaging for $\mathrm{NaI}$ than for $\mathrm{LiH}$ due to the larger number of initial $J$ states contributing; see Fig. 5. For NaI all initial states corresponding to $J \leq 16$ were included in the calculation. Again, an orientation effect is observed after turn-off of the pulse, with full revivals spaced by $\tau=$ 138 ps. When compared to the results for $\mathrm{LiH}$, it is clear that a higher field strength is required in order to induce a noticeable time-dependent orientation. The oscillations in the orientation parameter for $\mathrm{NaI}$ are "wiped out" at higher temperatures due to the less efficient orientation of the higher initial $J$ states. In addition, small deviations from rigid-rotor dynamics can play a role.

With the low field strengths investigated in this Letter, the orientation effect disappears when the pulse contains one or more full optical oscillations. In practice, in order to avoid pulses with very high intensities a pulse containing a single optical oscillation or half-cycle electromagnetic pulses are required.

The key result presented in this Letter is the prediction of field-free time-dependent orientation for a molecule like $\mathrm{LiH}$, using the current state-of-the-art HCP. Slightly higher field strengths will induce a very pronounced field-free time-dependent orientation which, in addition, is very robust towards thermal averaging.

This work was supported by the Danish Natural Science Research Council and The Carlsberg Foundation.

[1] Special issue on Stereodynamics of Chemical Reactions [J. Phys. Chem. A 101, No. 41 (1997)].

[2] H. Sakai, C. P. Safvan, J. J. Larsen, K. M. Hilligsøe, K. Held, and H. Stapelfeldt, J. Chem. Phys. 110, 10235 (1999).

[3] M. Machholm and N.E. Henriksen, J. Chem. Phys. 111, 3051 (1999).

[4] R. N. Zare, Ber. Bunsen-Ges. Phys. Chem. 86, 422 (1982).

[5] V. A. Cho and R. B. Bernstein, J. Phys. Chem. 95, 8129 (1991).

[6] B. Friedrich and D. R. Herschbach, Nature (London) 353, 412 (1991).

[7] H. J. Loesch and A. Remscheid, J. Phys. Chem. 95, 8194 (1991).

[8] B. Friedrich and D. R. Herschbach, Phys. Rev. Lett. 74, 4623 (1995).

[9] C. M. Dion, A. D. Bandrauk, O. Atabek, A. Keller, H. Umeda, and Y. Fujimura, Chem. Phys. Lett. 302, 215 (1999).

[10] T. Seideman, J. Chem. Phys. 103, 7887 (1995).

[11] T. Seideman, Phys. Rev. Lett. 83, 4971 (1999).

[12] J. Ortigoso, M. Rodriguez, M. Gupta, and B. Friedrich, J. Chem. Phys. 110, 3870 (1999).

[13] N. E. Henriksen, Chem. Phys. Lett. 312, 196 (1999).

[14] L. Cai, J. Marango, and B. Friedrich, Phys. Rev. Lett. 86, 775 (2001).

[15] D. You, R. R. Jones, P. H. Bucksbaum, and D. R. Dykaar, Opt. Lett. 18, 290 (1993).

[16] R. Kosloff, J. Phys. Chem. 92, 2087 (1988).

[17] H. Partridge and S. R. Langhoff, J. Chem. Phys. 74, 2361 (1981).

[18] G. H. Peslherbe, R. Bianco, J. T. Hynes, and B. M. Landanyi, J. Chem. Soc. Faraday Trans. 93, 977 (1997). 